\title{
Place de la simulation dans la formation initiale des urgentistes : enquête nationale observationnelle
}

\author{
Place of simulation in the initial training of french emergency physicians: national observational \\ and descriptive study
}

\author{
M. Allain - V. Kuczer - C. Longo - E. Batard - P. Le Conte \\ Reçu le 14 février 2018; accepté le 10 avril 2018 \\ (C) SFMU et Lavoisier SAS 2018
}

Résumé Introduction : La simulation apparaît aujourd'hui comme un outil indispensable dans l'apprentissage de compétences médicales techniques et non techniques, tout en respectant le principe éthique suivant : « Jamais la première fois sur le patient $»$. Actuellement, l'intégration de la simulation dans les programmes de formation des internes de médecine d'urgence est disparate : son cadre demeure à définir devant l'ouverture récente du diplôme d'études spécialisée (DES) de médecine d'urgence à l'automne 2017.

Objectif de l'étude : Cette étude a évalué l'utilisation de la simulation en France dans l'enseignement actuel de la médecine d'urgence, afin d'initier une réflexion sur la place que pourrait avoir ce type d'enseignement pour le DES.

Méthode : Il s'agissait d'une étude observationnelle multicentrique. Un questionnaire a été envoyé par e-mail à l'ensemble des responsables universitaires de France impliqués dans l'enseignement de la médecine d'urgence. Les données ont été colligées dans Excel (Microsoft). Elles étaient à la fois quantitatives et qualitatives et concernaient le cadre, la structuration et le contenu de la formation par la simulation.

Résultats : Vingt-cinq facultés de médecine sur 29 ont répondu au questionnaire. Parmi elles, 23 utilisaient la simulation (soit $92 \%$ ), dont 22 la simulation haute fidélité et 21 la simulation procédurale. Arrêt cardiorespiratoire (22), état de choc (21), douleur thoracique et tachycardies (20), trauma-

M. Allain $(\bowtie) \cdot$ V. Kuczer $\cdot$ C. Longo $\cdot$ P. Le Conte

Service des urgences-Samu 44, CHU de Nantes,

1, place Alexis-Ricordeau, F-44000 Nantes, France

e-mail : milena.allain@chu-nantes.fr

C. Longo $\cdot$ E. Batard

CESU 44, quai Moncousu, F-44093 Nantes, France

E. Batard · P. Le Conte

Faculté de médecine, université de Nantes,

1, quai de Tourville, F-44000 Nantes, France tisé crânien et traumatisé grave (17) étaient les thématiques majoritairement abordées. Les gestes techniques les plus enseignés étaient : intubation (22), intubation difficile (21), ventilation assistée-contrôlée et voie intraosseuse (18). En médiane, la formation comptait entre deux et trois jours de simulation par année de formation. Par ailleurs, dans 12 centres (soit $52 \%$ ), les formateurs avaient un temps professionnel dédié, et 15 centres (soit $65 \%$ ) bénéficiaient de personnel salarié. Enfin, seulement 12 centres (52\%) estimaient « plutôt » respecter l'adage « Jamais la première fois sur le patient $»$.

Discussion : L'utilisation de la simulation dans l'enseignement de la médecine d'urgence est inégale. Les responsables pédagogiques interrogés semblent tomber d'accord quant aux principales thématiques et techniques à aborder. Le développement de la simulation semble souhaité de tous, mais demeure complexe en raison notamment de l'investissement humain et matériel que cela représente. Ces réponses pourraient fournir un axe de réflexion afin d'établir un programme de simulation commun à l'ensemble des facultés.

Mots clés Simulation - Formation initiale $\cdot$ Médecine d'urgence $\cdot$ DESC $\cdot$ DES $\cdot$ France

Abstract Background: Simulation appears to be now an essential tool for the training of both technical and nontechnical medical skills. It allows to respect the ethical statement: "Never the first time on a real patient". Currently, integration of simulation in training programs is not harmonized between French medicine faculties. The corresponding framework remains to be defined in anticipation of the creation of the diploma of Emergency Medical Specialty (DES) planned for fall 2017.

Objective: This investigation addressed the use of simulation in French initial formation in emergency medicine. It was intended as a first step toward establishing a simulationbased training program. 
Method: This observational study was multicentric. A questionnaire was sent by e-mail to all coordinators of Emergency Medicine initial formation in France. Data were compiled with Excel software (Microsoft Inc.). They included both quantitative and qualitative items that concerned the general context, the structure, and the content of the simulation-based training.

Results: Twenty-five out of 29 universities answered the questionnaire. Among those, 23 (92\%) used simulation, 22 of them used high-fidelity simulation, and 21 used procedural simulation. The most involved application fields were: cardiac arrest (22), shock state (21), chest pain, tachycardia (20), severe trauma and traumatic brain injury (17). The most practiced technical procedures were: intubation (22), difficult intubation (21), mechanical ventilation and intraosseous access (18). Median time was 2-3 days of simulation by year. In 12 centers $(52 \%)$, the training staff had dedicated time duty and 15 centers $(65 \%)$ benefited from remunerated staff. Finally, only 12 centers $(52 \%)$ felt they essentially complied with the statement "Never the first time on a real patient".

Discussion: Simulation use in French emergency physician's initial training is heterogeneous. The main limitation of our study was the absence of response from 4 faculties. Generally, responding coordinators agreed on the main situations and techniques to be taught. As in other countries, promotion of simulation seems to be consensual. However, effective deployment remains difficult due to material investment, formation and availability of staff. This study could provide a basis for the establishment of a national simulation-based training program.

Keywords Simulation - Initial training - Emergency medicine $\cdot$ National study $\cdot$ DES

\section{Introduction}

La simulation professionnelle moderne est née de l'aéronautique au début $\mathrm{du} \mathrm{xx}^{\mathrm{e}}$ siècle, rapidement imitée dans le cadre des formations de la marine marchande et de l'industrie nucléaire, dans un objectif de sécurisation de ces domaines professionnels «à risque». La formation par la simulation en santé se développe intensément ces dernières années [1], en premier lieu, pour les médecins anesthésistes et depuis peu également en médecine d'urgence [2-4]. Cette technique apparaît complémentaire aux autres méthodes d'enseignement, et son cadre est désormais bien défini pour le Développement professionnel continu [5]. La simulation permet l'apprentissage de procédures standardisées, le développement de compétences techniques [6-8]. Elle est un outil pour l'acquisition de compétences humaines et comportementales nécessaires à l'exercice de notre profession [9-11]. Elle procure un environnement de travail sécurisé à la fois pour le patient et pour l'étudiant, dans l'apprentissage des procédures à risque $[12,13]$. La simulation offre un espace d'entraînement réaliste, dans lequel l'étudiant « apprend en faisant » [14] et peut devenir acteur de sa propre formation [15-18]. C'est un outil pédagogique permettant l'analyse de situations de travail, ainsi que la reconnaissance et le développement des compétences nécessaires pour résoudre ces situations.

En France, l'enseignement de la médecine d'urgence comme une discipline autonome s'individualise depuis les années 1980. Plusieurs diplômes universitaires se sont succédés : la Capacité d'aide médicale urgente (Camu) en 1986, remplacée par la Capacité de médecine d'urgence (CMU) en 1998, puis par le Diplôme d'études spécialisées complémentaires (DESC) de médecine d'urgence en 2004. À l'automne 2017, la création du Diplôme d'études spécialisées (DES) de médecine d'urgence a permis le développement académique et la reconnaissance de cette discipline comme une spécialité à part entière [19]. Cette évolution imposait une réflexion afin de répondre aux nouvelles exigences de formation. L'utilisation de la simulation dans la formation initiale des urgentistes n'a pas été étudiée. Son intégration aux curricula de médecine d'urgence doit être organisée et planifiée, au même titre que les enseignements théoriques.

L'objectif principal de cette étude était de réaliser un état des lieux des pratiques de simulation dans la formation proposée aux internes de DESC de médecine d'urgence, de préciser sa place, sa structuration, son organisation. Les objectifs secondaires étaient d'identifier les facteurs limitant le développement de cet outil pédagogique, d'initier une réflexion autour de la forme et du contenu que pourrait avoir la formation par simulation, dans le cadre du DES de médecine d'urgence.

\section{Méthode}

Il s'agissait d'une étude observationnelle descriptive nationale interrogeant l'utilisation de la simulation dans le cadre de la formation initiale des futurs urgentistes. Un questionnaire a été envoyé par courriel aux directeurs des centres de simulation et/ou aux responsables pédagogiques du DESC de médecine d'urgence des 29 villes universitaires françaises. Plusieurs relances ont été effectuées entre septembre 2016 et mars 2017.

Le premier volet du questionnaire, appelé «Questions générales ", interrogeait l'utilisation de la simulation dans le cadre de la formation du DESC de médecine d'urgence, l'année d'introduction de la simulation pour les internes de DESC, les raisons pouvant expliquer la non-utilisation de 
cette méthode. Le deuxième volet du questionnaire, intitulé «Questions relatives au centre de simulation et à son fonctionnement », interrogeait la structure, le type de simulation pratiqué, l'organisation et les équipements techniques du centre de simulation concerné. Le troisième volet du questionnaire concernait les « Questions relatives à la formation en elle-même ", à savoir : le caractère obligatoire de la formation, le nombre et la durée des séances, le nombre de scenarii par séance et leur répétition, l'organisation de chaque séance, les effectifs des groupes et leur composition, les objectifs pédagogiques, le type et la méthode d'évaluation. Le contenu de l'enseignement était interrogé dans cette partie du questionnaire par un listing de thématiques cliniques et de gestes techniques établi par les auteurs. Ce dernier volet du questionnaire se terminait par deux questions dans lesquelles le répondeur estimait la qualité du respect de l'adage « Jamais la première fois sur le patient » et précisait ses projets d'évolutivité de la formation par simulation.

\section{Analyse statistique}

Les données ont été colligées et analysées par le logiciel Excel 2016 (Microsoft, Redmond, WA, États-Unis). Les résultats sont donnés sous forme de pourcentages, médianes et moyennes. Les pourcentages sont accompagnés des effectifs correspondants. Les médianes et les moyennes sont suivies de leurs valeurs extrêmes (minimum [min] ; maximum $[\max ])$.

\section{Résultats}

Au total, nous avons obtenu 25 réponses sur 29 (86\%). Sur les 25 réponses obtenues, 23 responsables pédagogiques déclaraient utiliser la simulation dans la formation initiale des urgentistes $(92 \%)$. Parmi les deux facultés n'utilisant pas la simulation en formation initiale, une avait un centre de simulation non utilisé par les étudiants de DESC, une autre faculté n'avait pas de centre de simulation du tout. Au moment de notre étude, la plupart des facultés de France utilisaient cet outil pédagogique depuis 2011 en médiane $(\min =2004 ; \max =2016)$ pour la formation initiale des urgentistes.

Sur les 23 facultés interrogées, 13 (56\%) rapportaient que le statut du centre était mixte hospitalo-universitaire, sept (30\%) hospitalier, deux (8\%) universitaire. À noter que le statut du centre n'était pas un facteur d'exclusion de l'étude. Les techniques les plus utilisées étaient la simulation haute fidélité pour 22 facultés (96\%), procédurale pour 21 (91\%) et basse fidélité pour 18 (78\%). L'utilisation de patients standardisés et l'usage de jeux de rôles étaient respectivement retrouvés chez $14(61 \%)$ et $12(52 \%)$ facultés. Les autres types de simulation (jeux sérieux, réalité virtuelle, simulation « in situ ») étaient plus rarement pratiqués (Tableau 1). La majorité des centres pouvaient utiliser la vidéo (19, soit $83 \%$ ). Pour 15 centres $(65 \%)$, cet outil était utilisé pour la visualisation de la séance par les observateurs et comme outil pour le débriefing.

La composition des équipes pédagogiques était variable avec une médiane de cinq formateurs médicaux $(\min =2$; $\max =12$ ) et une médiane de 1,25 formateur paramédical $(\min =0 ; \max =9)$. La formation des enseignants était diverse, allant d'une formation de quelques jours aux techniques de simulation jusqu'au master en pédagogie en santé. De plus, 12 centres $(52 \%)$ déclaraient bénéficier de temps professionnel dédié à l'activité de simulation. En tout, 15 centres $(65 \%)$ étaient dotés de personnel salarié, en médiane une personne $(\min =0 ; \max =6)$.

La formation était déclarée « obligatoire » pour 18 facultés $(78 \%)$ et « base obligatoire + séances facultatives » pour cinq $(22 \%)$. Par ailleurs, 18 facultés $(78 \%)$ exprimaient avoir un objectif de formation seulement, quand cinq ( $22 \%)$ avaient en plus un objectif d'évaluation certificative. La durée des séances était variable, la majorité des facultés $(15$, soit $65 \%)$ organisaient leur formation sous forme de journées. Pour l'ensemble des facultés, une médiane de deux jours de simulation était prévue en première année de DESC $(\min =0 ; \max =18)$, de trois jours en deuxième année $(\min =1 ; \max =18)$. La moyenne du nombre de scenarii par séance était de quatre $(\min =2$; $\max =7$ ).

Les thèmes abordés en simulation haute fidélité considérés comme « très fréquents » (17 facultés ou plus, soit $75 \%$ de l'échantillon ou plus) étaient : l'arrêt cardiorespiratoire ; les états de choc ; les douleurs thoraciques ; les tachycardies ; les traumatisés crâniens ; les traumatisés graves (Tableaux 2-4).

Tableau 1 Types de simulation utilisés dans le cadre de la formation initiale des urgentistes $(n=23)$

\begin{tabular}{|ll|}
\hline Type de simulation & Nombre de facultés (\%) \\
\hline Simulation haute fidélité & $22(96)$ \\
Simulation procédurale & $21(91)$ \\
Simulation basse fidélité & $18(78)$ \\
Patient standardisé & $14(61)$ \\
Jeux de rôles & $12(52)$ \\
Simulation « in situ » & $8(35)$ \\
Jeux sérieux & $7(30)$ \\
Réalité virtuelle & $6(26)$ \\
Autre type de simulation & $1(4)$ \\
Non précisé & $1(4)$ \\
\hline Résultats exprimés en nombre (\%) sur effectif total \\
de 23 facultés
\end{tabular}




\begin{tabular}{|c|c|}
\hline Thématique enfant & Nombre de facultés $(\%)$ \\
\hline \multicolumn{2}{|c|}{ Prise en charge du nouveau-né } \\
\hline Situation physiologique & $8(35)$ \\
\hline Situation pathologique & $3(12)$ \\
\hline Convulsions & $6(26)$ \\
\hline Détresse respiratoire & $11(48)$ \\
\hline Purpura fulminans & $5(22)$ \\
\hline Arrêt cardiorespiratoire & $12(52)$ \\
\hline Traumatisé grave & $5(22)$ \\
\hline \multicolumn{2}{|c|}{$\begin{array}{l}\text { Résultats exprimés en nombre }(\%) \text { sur effectif tota } \\
\text { de } 23 \text { facultés }\end{array}$} \\
\hline
\end{tabular}

Les gestes et techniques abordés en simulation procédurale considérés comme « très fréquents » étaient : l'intubation orotrachéale ; l'intubation difficile ; la ventilation mécanique assistée contrôlée ; la pose de voie intraosseuse ; la ventilation non invasive (Tableau 5). Treize facultés (56\%) ne pratiquaient pas de répétition de scenarii, quand dix (44\%) déclaraient en faire.

Parmi les facultés interrogées, 14 (61\%) déclaraient qu'un prérequis théorique était exigé, et 15 (65\%) réalisaient un point théorique sur le sujet abordé en cours de séance. Un briefing général de début de séance était pratiqué par 19 centres (83\%), 17 (74\%) effectuaient un briefing avant chaque scénario, un $(4 \%)$ ne pratiquait ni l'un ni l'autre. Par ailleurs, 22 facultés (96\%) réalisaient un débriefing après chaque scénario, seulement huit $(35 \%)$ réalisaient un débriefing général de fin de séance, et une (4\%) déclarait ne pas pratiquer de débriefing. Concernant l'évaluation de fin de séance, 19 facultés ( $83 \%$ ) déclaraient en effectuer une. La satisfaction des étudiants était évaluée par 17 facultés $(74 \%)$, les acquis théoriques en cours de formation par huit (35\%). Cette évaluation était réalisée à l'aide d'échelles d'évaluation pour 14 facultés $(61 \%)$ et avec une fiche d'aide à la progression pour trois $(12 \%)$. Les effectifs étaient de moins de dix étudiants pour 14 facultés $(61 \%)$, et de 10 à 20 étudiants pour huit facultés (35\%). La formation était adressée exclusivement aux internes de DESC de médecine d'urgence pour huit facultés (35\%), 12 (52\%) signalaient des groupes mixtes comprenant des professionnels paramédicaux, neuf (39\%) avec médecins, trois (12\%) avec internes de spécialités. À la question : «Diriez-vous que la formation actuelle dans votre faculté permet le "jamais la première fois sur le patient" ? ", 12 facultés $(48 \%)$ répondaient " plutôt oui », 11 (44\%) répondaient « plutôt non », et aucune faculté n'optait pour l'option « oui, tout à fait».
Tableau 3 Thématiques abordées en simulation moyenne à haute fidélité chez l'adulte — partie $1(n=23)$

\begin{tabular}{|c|c|}
\hline Thématique adulte & $\begin{array}{l}\text { Nombre } \\
\text { de facultés (\%) }\end{array}$ \\
\hline \multicolumn{2}{|l|}{ Cardiologie } \\
\hline Arrêt cardiorespiratoire & $22(96)$ \\
\hline ECMO & $2(8)$ \\
\hline DDAC & $1(4)$ \\
\hline Douleur thoracique & $20(87)$ \\
\hline SCA & $16(69)$ \\
\hline Diagnostics différentiels du SCA & $8(35)$ \\
\hline $\begin{array}{l}\text { Tachycardies ventriculaires } \\
\text { et supraventriculaires }\end{array}$ & $20(87)$ \\
\hline Troubles de la conduction intracardiaque & $16(69)$ \\
\hline Embolie pulmonaire & $12(52)$ \\
\hline États de choc & $21(91)$ \\
\hline \multicolumn{2}{|l|}{ Respiratoire } \\
\hline Asthme aigu grave & $14(61)$ \\
\hline Décompensation de BPCO & $12(52)$ \\
\hline OAP & $16(69)$ \\
\hline Pneumothorax & $15(65)$ \\
\hline Épanchement pleural & $7(30)$ \\
\hline $\begin{array}{l}\text { Obstruction des voies aériennes } \\
\text { supérieures }\end{array}$ & $10(43)$ \\
\hline \multicolumn{2}{|l|}{ Neurologie } \\
\hline Coma non traumatique & $13(56)$ \\
\hline Vasculaire & $5(22)$ \\
\hline Toxique & $9(39)$ \\
\hline Métabolique & $5(22)$ \\
\hline État de mal épileptique & $14(61)$ \\
\hline \multicolumn{2}{|l|}{ Gastroentérologie et urologie } \\
\hline Hémorragie digestive & $4(17)$ \\
\hline \multicolumn{2}{|l|}{ Infectiologie } \\
\hline Accès palustre & $0(0)$ \\
\hline Purpura fulminans et méningoencéphalite & $6(26)$ \\
\hline $\begin{array}{l}\text { Résultats exprimés en nombre }(\%) \text { sur effecti } \\
\text { tés. ECMO : extracorporelle membrane oxyg } \\
\text { donneur d'organes décédé après arrêt cardia } \\
\text { drome coronarien aigu ; BPCO : bronchopr } \\
\text { nique obstructive ; OAP : œdème aigu pulm }\end{array}$ & $\begin{array}{l}\text { f total de } 23 \text { facul- } \\
\text { génation ; DDAC : } \\
\text { aque ; SCA : syn- } \\
\text { neumopathie chro- } \\
\text { onaire }\end{array}$ \\
\hline
\end{tabular}

\section{Discussion}

L'utilisation de la simulation en formation initiale de médecine d'urgence concerne la majorité des facultés françaises. L'absence totale de centre dans une faculté française prouve cependant que la diffusion de cette méthode d'enseignement peut encore progresser. La médecine d'urgence est, tout comme les domaines du nucléaire et de l'aéronautique, une discipline à haut risque. Le risque d'erreur est inhérent à la 
Tableau 4 Thématiques abordées en simulation moyenne à haute fidélité chez l'adulte — partie $2(n=23)$

\begin{tabular}{|c|c|}
\hline Thématique adulte & $\begin{array}{l}\text { Nombre } \\
\text { de facul }\end{array}$ \\
\hline \multicolumn{2}{|l|}{ Troubles électrolytiques et métaboliques } \\
\hline Hypoglycémie & $7(30)$ \\
\hline Hyperglycémie et acidocétose diabétique & $3(12)$ \\
\hline Trouble ionique & $7(30)$ \\
\hline \multicolumn{2}{|l|}{ Gynécologie et obstétrique } \\
\hline Accouchement inopiné & $12(52)$ \\
\hline Éclampsie et prééclampsie & $3(12)$ \\
\hline Hémorragie de la grossesse, GEU & $3(12)$ \\
\hline Hémorragie de la délivrance & $6(26)$ \\
\hline \multicolumn{2}{|l|}{ Traumatologie } \\
\hline $\begin{array}{l}\text { Traumatisme crânien avec signe } \\
\text { de localisation/coma }\end{array}$ & $17(74)$ \\
\hline $\begin{array}{l}\text { Traumatisme du rachis avec déficit } \\
\text { neurologique }\end{array}$ & $8(35)$ \\
\hline Traumatisé grave & $17(74)$ \\
\hline Autres traumatismes & $12(52)$ \\
\hline Fracture du bassin & $6(26)$ \\
\hline Fracture de membre & $6(26)$ \\
\hline Traumatisme thoracique & $6(26)$ \\
\hline Plaie hémorragique & $8(35)$ \\
\hline Plaie balistique & $4(17)$ \\
\hline Brûlure grave & $4(17)$ \\
\hline \multicolumn{2}{|l|}{ Pathologies circonstancielles } \\
\hline Noyade & $1(4)$ \\
\hline Électrisation & $1(4)$ \\
\hline Pendaison & $7(30)$ \\
\hline Hypothermie-hyperthermie & $0(0)$ \\
\hline \multicolumn{2}{|l|}{ Divers } \\
\hline Accident des anticoagulants & $4(17)$ \\
\hline $\begin{array}{l}\text { Prise en charge multivictimes, Orsec- } \\
\text { Novi, attentat }\end{array}$ & $4(17)$ \\
\hline NRBC & $2(8)$ \\
\hline $\begin{array}{l}\text { Consultation d'annonce de pathologie } \\
\text { grave, de décès }\end{array}$ & $1(4)$ \\
\hline Simulation de régulation médicale & $8(35)$ \\
\hline $\begin{array}{l}\text { Utilisation de l'échographie intégrée au } \\
\text { scénario }\end{array}$ & $8(35)$ \\
\hline
\end{tabular}

Résultats exprimés en nombre (\%) sur effectif total de 23 facultés. GEU : grossesse extra-utérine ; Orsec-Novi : Organisation de la réponse de sécurité civile-Nombreuses victimes ; NRBC : nucléaire radiologique biologique chimique

diversité des activités pratiquées aux urgences et au grand nombre de patients vus dans une temporalité stricte. La simulation est un moyen d'améliorer la sécurité des soins en procurant un environnement de travail sûr. Son intégra-
Tableau 5 Gestes et techniques d'urgence abordés en simulation procédurale $(n=23)$

\begin{tabular}{|ll|}
\hline Geste ou technique d'urgence & $\begin{array}{l}\text { Nombre } \\
\text { de facultés (\%) }\end{array}$ \\
\hline IOT & $22(96)$ \\
IOT difficile & $21(91)$ \\
VAC & $18(78)$ \\
VNI & $17(74)$ \\
Exsufflation à l'aiguille du pneumothorax & $13(56)$ \\
Pose de drain thoracique & $12(52)$ \\
Ponction péricardique & $0(0)$ \\
Toucher vaginal & $3(12)$ \\
Manœuvres de l'accouchement & $12(52)$ \\
Pose de cathéter intraosseux & $18(78)$ \\
Pose de voie centrale & $9(39)$ \\
Pose de cathéter intraombilical & $1(4)$ \\
BIF & $7(30)$ \\
Ponction lombaire & $2(8)$ \\
Ponction d'ascite & $0(0)$ \\
Ponction articulaire & $0(0)$ \\
Cathéter sus-pubien & $0(0)$ \\
Stratégies de damage control & $5(22)$ \\
Autotransfusion & $0(0)$ \\
Échographie appliquée à l'urgence & $10(43)$ \\
Utilisation du défibrillateur & $15(65)$ \\
Pose de SEES & $5(22)$ \\
\hline Résultats exprimés en nombre (\%) sur effectif total de 23 facul- \\
tés. IOT : intubation orotrachéale ; VAC : ventilation mécanique \\
assistée contrôlée ; VNI : ventilation non invasive ; BIF : bloc \\
iliofacial & \\
a Reconnaissance du degré de dilatation du col utérin en travail \\
\hline
\end{tabular}

tion dans les programmes doit être sans équivoque, elle est évoquée dans les arrêtés de novembre 2015 et avril 2017 du ministère de l'Éducation nationale [20,21].

Le statut des centres de simulation participant à la formation initiale était hospitalo-universitaire pour la moitié des facultés. Il apparaît indispensable de développer des liens entre l'université et l'hôpital afin d'assurer le bon fonctionnement des centres de simulation $[1,12,22]$. Le type de simulation utilisé dépend des moyens financiers et de la taille des locaux. Les mannequins basse fidélité reproduisent tout ou partie du corps humain et sont statiques. Parmi eux, les simulateurs procéduraux sont spécialement conçus pour l'apprentissage d'un geste technique (bras pour perfusion, tête d'intubation, etc.). Les mannequins haute fidélité ont une interface informatique complexe. Proches du réel, ils reproduisent de nombreux paramètres vitaux, signes cliniques et permettent la réalisation de gestes. Le patient standardisé est un acteur 
formé pour simuler une situation clinique. Les techniques de réalité virtuelle sont des programmes informatiques au travers desquels l'étudiant peut interroger et examiner un patient. Parmi ces techniques, les jeux sérieux sont des modules proches de jeux vidéo dans lesquels le médecin apprend à gérer des situations cliniques. Enfin, la simulation « in situ » est une simulation pratiquée dans l'environnement de travail habituel. Le choix des outils utilisés doit être guidé par les objectifs pédagogiques et non répondre à une recherche de technologie de pointe « à tout prix » [12]. Les outils de simulation procédurale et de haute fidélité (les plus cités) sont complémentaires et semblent en effet indispensables.

Par ailleurs, travailler dans un centre de simulation demande un investissement important. La reconnaissance du temps professionnel consacré à la simulation est un objectif incontournable si nous souhaitons l'évolution de cette modalité pédagogique. Des disparités sont mises en évidence au sein des équipes pédagogiques dans cette étude. La formation des formateurs manquait d'uniformisation sur le territoire, alors que celle-ci fait partie des recommandations de bonnes pratiques [12]. La présence de personnel salarié, condition pourtant nécessaire au bon fonctionnement d'une telle structure, était inégale.

Les disponibilités des enseignants et des locaux mises à part, le nombre de séances nécessaire dépend du contenu pédagogique établi ; il n'a pas été discuté dans la littérature à ce jour. La médiane nationale rapportée par cette étude nous semble être un premier objectif raisonnable. De même, la durée optimale d'une séance de simulation n'a pas été démontrée dans la littérature. Une séance de simulation correspond à un enchaînement de plusieurs phases. Le briefing est l'étape préparatoire pendant laquelle sont rappelés les objectifs, les règles déontologiques et de confidentialité. Le formateur tente de créer un climat favorable pour l'apprentissage, les apprenants font connaissance avec le matériel et l'environnement. Pendant le prébrief, le formateur prépare les apprenants à l'exercice de simulation, contextualise la situation clinique du scénario. Vient juste après la phase de pratique simulée : certains étudiants « jouent » pendant que les autres observent. Après la simulation est réalisé le débriefing, phase la plus importante sur le plan pédagogique. Son objectif est d'optimiser l'apprentissage en stimulant la réflexivité des apprenants. L'exercice de « contextualisation-décontextualisation » des connaissances permet leur généralisation à la pratique réelle [12,23-25]. Ce cheminement est essentiel pour améliorer les compétences et, à terme, les performances cliniques. Le débriefing doit, selon la Haute Autorité de santé (HAS), au moins être égal au temps consacré à la pratique simulée [1]. Notons que l'unité « prébrief ; pratique simulée ; débriefing » peut se répéter plusieurs fois au cours de la séance (plusieurs scenarii successifs), avant une phase de conclusion. Par ailleurs, la répétition de scenarii était selon cette étude loin d'être systématique malgré les recommandations. Les méthodes d'évaluation utilisées en France étaient diverses et mériteraient une uniformisation.

En ce qui concerne les effectifs des groupes d'étudiants, il semble important que ceux-ci soient relativement restreints, afin de pouvoir expérimenter successivement les positions d'acteur et d'observateur. Le ratio enseignant/étudiants doit être raisonnable pour que les formateurs soient en mesure d'apporter un feed-back constructif et personnalisé à chaque étudiant. Ce ratio n'a pas été discuté dans la littérature en ce qui concerne la simulation en santé. Par ailleurs, le développement de séances mixtes associant du personnel paramédical aux étudiants semble souhaité par de nombreuses facultés, bien que ne concernant que moins de la moitié d'entre elles au moment de l'étude. Ce type de séance favorise en effet l'acquisition de compétences relatives au travail en équipe $[9,10]$ et représente un enjeu organisationnel pour l'avenir. L'utilisation de la simulation comme outil d'évaluation certificative est interrogée depuis plusieurs années, bien implantée dans certains pays comme les États-Unis $[5,26,27]$. Au moment de notre étude, peu de facultés françaises s'étaient engagées dans cette voie.

Les thématiques et gestes techniques que nous avons classés dans la catégorie « très fréquents » sont (pour la plupart) largement étudiés dans la littérature [8,11,28-30]. Certains sujets moins abordés selon cette étude mériteraient cependant d'être travaillés en simulation. La régulation médicale, acte complexe et faisant partie intégrante de notre activité professionnelle, pourrait être enseignée davantage. L'enseignement de l'échographie en médecine d'urgence doit aussi être questionné. Son intégration en simulation paraît indispensable afin de se rapprocher le plus possible de l'exercice clinique quotidien. Devant les événements récents, il nous semble également essentiel de proposer une expérience simulée dans le domaine des situations sanitaires exceptionnelles : dispositif Orsec-Novi (Organisation de la réponse de sécurité civile-Nombreuses victimes), plan blanc, techniques de damage control.

Enfin, encore trop peu de facultés considéraient au moment de notre étude respecter l'adage éthique porté par la simulation en santé : "Jamais la première fois sur le patient $»$. L'usage de la simulation ne dispense aucun médecin de sa véritable première expérience auprès du malade qui doit elle aussi pouvoir s'effectuer dans un cadre sécurisé.

La principale limite de notre étude était l'absence de réponse de quatre facultés sur les 29 interrogées. Le recueil des données a été effectué uniquement sur les données déclaratives du questionnaire. Cette méthode ne permettait pas d'effectuer une évaluation objective des performances techniques et non techniques des étudiants en formation ni une évaluation des compétences pédagogiques des formateurs. Le listing des thématiques cliniques et des gestes techniques 
était rédigé par les auteurs et se fondait sur leur expérience clinique personnelle et non sur un référentiel reconnu.

\section{Conclusion}

La simulation a pris une place considérable dans l'enseignement initial des urgentistes, son intérêt pédagogique n'est plus à démontrer. Son intégration demeure cependant hétérogène sur le territoire français. L'élaboration d'une formation par simulation exige une grande rigueur et doit être guidée par les règles de bonne pratique établies par la HAS. La recherche doit se poursuivre afin de préciser les critères de qualité d'une telle formation en médecine d'urgence. Le développement de moyens humains et matériels, l'uniformisation de la formation des formateurs en simulation, la reconnaissance du temps professionnel qui en découle représentent des enjeux incontournables si nous souhaitons voir évoluer la simulation en médecine d'urgence. La création du DES de médecine d'urgence est l'occasion de réformer la formation par simulation et de lui accorder la place qu'elle mérite dans l'enseignement de l'urgence.

Conflits d'intérêts : Les auteurs déclarent ne pas avoir de liens d'intérêts.

\section{Références}

1. Haute Autorité de santé (2012) Évaluation et amélioration des pratiques. Guide de bonnes pratiques en matière de simulation en santé. https://www.has-sante.fr/portail/upload/docs/application/pdf/ 2013-01/guide_bonnes_pratiques_simulation_sante_guide.pdf (Dernier accès le 29 mars 2018)

2. Gaba DM, Howard SK, Fish KJ, et al (2001) Simulation-based training in Anesthesia Crisis Resource Management (ACRM): a decade of experience. Simulat Gaming 32:175-95

3. Issenberg SB, McGaghie WC, Hart IR, et al (1999) Simulation technology for health care professional skills training and assessment. JAMA 282:861-66

4. Okuda Y, Bond W, Bonfante G, et al (2008) National growth in simulation training within emergency medicine residency programs, 2003-2008. Acad Emerg Med 15:1113-16

5. Granry JC, Moll MC (2012) Haute Autorité de santé. Rapport de mission. État de l'art (national et international) en matière de pratiques de simulation dans le domaine de la santé. https://www. has-sante.fr/portail/upload/docs/application/pdf/2012-01/simulation_en_sante_-_rapport.pdf (Dernier accès le 29 mars 2018)

6. Morgan PJ, Cleave-Hogg D, DeSousa S, et al (2004) Highfidelity patient simulation: validation of performance checklists. Br J Anaesth 92:388-92

7. Nguyen HB, Daniel-Underwood L, Van Ginkel C, et al (2009) An educational course including medical simulation for early goaldirected therapy and the severe sepsis resuscitation bundle: an evaluation for medical student training. Resuscitation 80:674-79

8. Jabre P, Jbeili C, Combes X, et al (2011) Intérêt de la simulation haute fidélité dans l'évaluation de l'application de procédures de soins par les médecins urgentistes. Ann Fr Med Urgence $1: 379-86$
9. Shapiro MJ, Gardner R, Godwin SA, et al (2008) Defining team performance for simulation-based training: methodology, metrics, and opportunities for emergency medicine. Acad Emerg Med 15:1089-97

10. Patterson PD, Weaver M, Weaver SJ, et al (2012) Measuring teamwork and conflict among emergency medical technician personnel. Prehosp Emerg Care 16:98-108

11. Harvey A, Nathens AB, Bandiera G, et al (2010) Threat and challenge: cognitive appraisal and stress responses in simulated trauma resuscitations. Med Educ 44:587-94

12. Boet S, Granry JC, Savoldelli G (2013) La simulation en santé, de la théorie à la pratique. Springer, Paris

13. Institute of Medicine (US) Committee on Quality of Health Care in America (2000) Kohn LT, Corrigan JM, Donaldson MS (editors) To err is human: building a safer health system. Institute of medicine. National Academy of Sciences. Washington (DC): National Academy Press:180-81

14. Levraut J, Fournier JP (2012) Jamais la première fois sur le patient ! Ann Fr Med Urgence 2:361-63

15. Giroux M, Girard G (2009) Favoriser l'apprentissage grâce à l'interaction superviseur-supervisé. Pedag Med 10:193-210

16. Schwartz LR, Fernandez R, Kouyoumjian SR, et al (2007) A randomized comparison trial of case-based learning versus human patient simulation in medical student education. Acad Emerg Med 14:130-37

17. McCoy CE, Menchine M, Anderson C, et al (2011) Prospective randomized crossover study of simulation vs. didactics for teaching medical students the assessment and management of critically ill patients. J Emerg Med 40:448-55

18. Owen H, Mugford B, Follows V, et al (2006) Comparison of three simulation-based training methods for management of medical emergencies. Resuscitation 71:204-11

19. Riou B (2017) 2017 : création du CNU de médecine d'urgence. Ann Fr Med Urgence 7:149-50

20. Ministère de l'Éducation nationale (2015) Arrêté du 13 novembre 2015 fixant la liste des diplômes d'études spécialisées de médecine. https://www.legifrance.gouv.fr/affichTexte.do?cidTexte=JORFTEXT000031560595\&categorieLien=id (Dernier accès le 29 mars 2018)

21. Ministère de l'Éducation nationale (2017) Arrêté du 21 avril 2017 relatif aux connaissances, aux compétences et aux maquettes de formation des diplômes d'études spécialisées et fixant la liste de ces diplômes et des options et formations spécialisées transversales du troisième cycle des études de médecine. https:// www.legifrance.gouv.fr/eli/arrete/2017/4/21/MENS1712264A/jo/ texte/fr (Dernier accès le 29 mars 2018)

22. Haute Autorité de santé (2015) Sécurité du patient, gérer les risques. Guide pour l'évaluation des infrastructures de simulation en santé. https://www.has-sante.fr/portail/upload/docs/application/ pdf/2015-07/guide pour_levaluation_des_infrastrures_de_simulation_en_sante_2015-07-21_11-26-51_939.pdf (Dernier accès le 6 avril 2018)

23. Fanning RM, Gaba DM (2007) The role of debriefing in simulation-based learning. Simul Healthc 2:115-25

24. Issenberg SB, McGaghie WC, Petrusa ER, et al (2005) Features and uses of high-fidelity medical simulations that lead to effective learning: a BEME systematic review. Med Teach 27: $10-28$

25. Boet S, Bould MD, Bruppacher HR, et al (2011) Looking in the mirror: self-debriefing versus instructor debriefing for simulated crises. Crit Care Med 39:1377-81

26. Yee B, Naik VN, Joo HS, et al (2005) Nontechnical skills in anesthesia crisis management with repeated exposure to simulation-based education. Anesthesiology 103:241-48 
27. Boulet JR (2008) Summative assessment in medicine: the promise of simulation for high stakes evaluation. Acad Emerg Med $15: 1017-24$

28. Wiel E, Lebuffe G, Erb C, et al (2009) Intérêt de la simulation réaliste dans l'évaluation de l'enseignement de l'intubation difficile aux médecins urgentistes. Ann Fr Anesth Reanim 28:542-48
29. Nguyen L, Jabre P, Monribot M, et al (2011) Courbe d'apprentissage et maintien des compétences pour la cricothyroïdotomie réalisée par des médecins urgentistes : étude sur mannequin. Ann Fr Med Urgence 1:91-5

30. Oriot D, Darrieux E, Boureau-Voultoury A, et al (2012) Validation of a performance assessment scale for simulated intraosseous access. Simul Healthc 7:171-77 